\title{
Theory and practice of natural computing: seventh edition
}

\author{
Carlos Martín-Vide ${ }^{1} \cdot$ Miguel A. Vega-Rodríguez $^{2}$
}

Published online: 5 January 2021

(C) The Author(s), under exclusive licence to Springer-Verlag GmbH, DE part of Springer Nature 2021

This special issue of the journal Soft Computing contains extended versions of some of the best papers presented at the Seventh International Conference on the Theory and Practice of Natural Computing, TPNC 2018, held in Dublin on December 12-14, 2018. The conference was co-organized by the Natural Computing Research \& Applications Group, School of Business, University College Dublin, and the Institute for Research Development, Training and Advice, Brussels/London.

TPNC 2018 was the seventh event in a series dedicated to presenting and promoting research on the wide spectrum of computational principles, models, and techniques inspired by information processing in nature. We intended to attract both theoretical and applied contributions to nature-inspired models of computation, synthesizing nature by means of computation, nature-inspired materials, and information processing in nature.

Out of 69 submissions to the conference, 35 papers were accepted (which represents an acceptance rate of about $51 \%$ ). Among them, the authors of 14 papers were invited to submit to this special issue. Each submission was reviewed by three experts and, based on their comments, the guest editors decided to accept 4 papers (which represents an acceptance rate of about $6 \%$ out of the submissions to the conference).

Next, we briefly present the papers included in this special issue.

In the paper Characterization and Computation of Ancestors in Reaction Systems, Roberto Barbuti, Anna Bernasconi, Roberta Gori, and Paolo Milazzo tackle the

Miguel A. Vega-Rodríguez

mavega@unex.es

Carlos Martín-Vide

carlos.martin@urv.cat

1 Research Group on Mathematical Linguistics, Rovira i Virgili University, 43002 Tarragona, Spain

2 ARCO Research Group, University of Extremadura, 10003 Cáceres, Spain computational complexity of preimages and nth ancestors in reaction systems. Despite the intractability of many of the problems, the authors find a Boolean formula computable in polynomial time that, once duly simplified, allows solving ancestor problems for classes of reaction systems in polynomial time.

Gaussian-kernel c-means Clustering Algorithms, by Shou-Jen Chang-Chien, Yessica Nataliani, and Miin-Shen Yang, deal with algorithms for clustering for datasets in a noisy environment or with diverse shape clusters. The authors use Gaussian kernels to generalize known AHCM and AFCM algorithms. Experimental results and comparisons show that the new algorithms improve the performance of previous ones.

Esma Mansouri-Benssassi and Juan Ye, in their paper Generalisation and Robustness Investigation for Facial and Speech Emotion Recognition Using Bio-inspired Spiking Neural Networks, approach the problems of robustness and generalizability of state-of-the-art techniques to recognize emotion in facial expressions and speech. They propose a spiking neural network model and evaluate it for accuracy with publicly available datasets. The results demonstrate better resistance to noise and more effective learning of feature representations in front of cross-datasets.

The paper Minor-embedding Heuristics for Largescale Annealing Processors with Sparse Hardware Graphs of up to 102,400 Nodes, by Yuya Sugie, Yuki Yoshida, Normann Mertig, Takashi Takemoto, Hiroshi Teramoto, Atsuyoshi Nakamura, Ichigaku Takigawa, Shin-ichi Minato, Masanao Yamaoka, and Tamiki Komatsuzaki, raises the question whether current embedding heuristics perform well on hardware graphs of very large size. The authors evaluate the performance of their improved version of a well-known annealing embedding heuristic on graphs of increasing size and find substantial improvements. They also prove some theoretical results on embeddability of complete graphs.

We thank you the authors for their contributions, the reviewers for their valuable work, and the editorial team 
of the journal for their professional support and collaboration.

\section{Compliance with ethical standards}

Conflict of interest The authors declare that they have no known competing financial interests or personal relationships that could have appeared to influence the work reported in this paper.
Publisher's Note Springer Nature remains neutral with regard to jurisdictional claims in published maps and institutional affiliations. 